\title{
Management strategies of neonatal jaundice during the coronavirus disease 2019 outbreak
}

\author{
Xiao-Lu Ma ${ }^{1,2} \cdot$ Zheng Chen ${ }^{1,2} \cdot$ Jia-Jun Zhu ${ }^{3} \cdot$ Xiao-Xia Shen ${ }^{1,2} \cdot$ Ming-Yuan Wu ${ }^{3} \cdot$ Li-Ping Shi $^{1,2} \cdot$ Li-Zhong Du $^{1,2}$. \\ Jun-Fen $\mathrm{Fu}^{1,2} \cdot$ Qiang Shu ${ }^{1,2}$
}

Received: 16 February 2020 / Accepted: 18 February 2020 / Published online: 28 February 2020

(c) Children's Hospital, Zhejiang University School of Medicine 2020

\begin{abstract}
The outbreak of coronavirus disease 2019 (COVID-19; formally known as 2019-nCoV) has become a most challenging health emergency. Owing to rigorous quarantine and control measures taken in China, routine neonatal health surveillance and follow-up have become challenging. Without follow-up surveillance, some rapid and progressive newborn diseases, such as bilirubin encephalopathy, may be ignored. The characteristics of onset age of kernicterus suggest that monitoring of bilirubin level at home provides a useful way to alert hospital visits and to prevent the development of extremely hyperbilirubinemia. Therefore, we developed an online follow-up program for convenient monitoring of bilirubin level of newborns that is based on our practical experiences. The aim is to make our management strategies of neonatal jaundice tailored to the infection prevention and control during the COVID-19 epidemic.
\end{abstract}

Keywords New coronavirus pneumonia $\cdot$ Hyperbilirubinemia $\cdot$ Newborn $\cdot$ Follow-up $\cdot$ Internet hospital

\section{Introduction}

The outbreak of coronavirus disease 2019 (COVID-19; formally known as 2019-nCoV), has become a most challenging health emergency [1,2]. Based on the current epidemiological and clinical evidences, the incubation period in pediatric patients ranges from 1 to 14 days [3]. So far, no documented cases of vertical transmission from mother to fetus have been reported [4]; however, mild-to-moderate respiratory distress, fever, thrombocytopenia, increased transaminase, and other non-specific presentations in neonates with negative tests who were born to infected mother warranted further studies on vertical transmission [5].

Jun-Fen Fu

fjf68@zju.edu.cn

Qiang Shu

shuqiang@zju.edu.cn

1 Children's Hospital, Zhejiang University School of Medicine, Hangzhou 310052, China

2 National Clinical Research Center for Child Health, National Children's Regional Medical Center, Hangzhou 310052, China

3 Women's Hospital, Zhejiang University School of Medicine, Hangzhou 310003, China
Owing to rigorous quarantine and control measures taken in China, routine neonatal health surveillance and follow-up have become challenging. Women and children are vulnerable to COVID-19 infection, so avoidance of routine hospital visits is recommended by the National Health Commission of China. However, delayed treatment of rapid and progressive newborn diseases can occur. Neonatal out-patient visits are of utmost important to those with hyperbilirubinemia. For unrecognized and accelerated severe early neonatal hyperbilirubinemia, appropriate interventions are utmost important to reduce the risks of neurological dysfunction, kernicterus, or even death. From a national survey in neonates in China in 2009, 348 cases of kernicterus were reported among 33 hospitals. An important finding of the study is that these patients presented with symptoms around ages of 5-6 days, and were mostly at home [6]. The characteristics of onset age of kernicterus suggest that monitoring of bilirubin level at home is a useful way to alert hospital visit and to prevent the development of extreme hyperbilirubinemia.

Therefore, we developed an online follow-up program for convenient monitoring of bilirubin level of newborns that is based on our practical experiences. The aim is to make our management strategies of neonatal jaundice tailored to the infection prevention and control during the COVID-19 epidemic. 


\section{Management strategies during hospitalization}

\section{Risk factor screening for severe hyperbilirubinemia}

Risk factors of severe hyperbilirubinemia include preterm birth, perinatal asphyxia, sepsis, metabolic acidosis, isoimmune hemolysis, glucose-6-phosphate dehydrogenase (G-6-PD) deficiency, cephalohematoma, significant skin bruising, hypoalbuminemia, jaundice observed in the first 24 hours, and exclusive breastfeeding with excessive weight loss [7].

Maternal medical history and birth history should be reviewed in detail. Every newborn infant should be examined comprehensively. According to the history, physical examination and risk factors, necessary diagnostic investigations, and laboratory tests (total serum bilirubin, count of blood cell, hematocrit, liver function, blood type, Coomb's test, G-6-PD screening, head ultrasound, etc.) were chosen to explore the underlying conditions.

\section{Early establishment of breastfeeding}

For infants without contraindications of enteral feeding, breastfeeding should be started at 20-30 min after birth. Infant's weight is measured every 1-2 days. Breastfed infants usually reach the maximum weight loss by day 3 of age, and most lose weight less than $10 \%$ of their birth weight. If the weight loss is more than $10 \%$, the adequacy of milk intake should be evaluated. Other evidence of adequate intake includes 4-6 thoroughly wet diapers in $24 \mathrm{~h}$ and the passage of 3-4 stools per day by the fourth day [7]. Medical staff should evaluate mother's lactation skills every day and identify the risks of inadequate breast milk intake promptly.

\section{Monitoring and management of hyperbilirubinemia before discharge}

Jaundice is usually seen first in the face of the infant, and then progresses to the trunk and extremities. Since the visual estimation of the degree of jaundice is not reliable, we recommend transcutaneous bilirubin (TCB) measurement as the first choice. The interval between two measurements is $12-24 \mathrm{~h}$. The results should be recorded in the chart. TCB is correlated with total serum bilirubin (TSB) decreasing as serum bilirubin concentration higher than $255 \mu \mathrm{mol} / \mathrm{L}$ (15 mg/dL), so TSB should be checked to confirm when TCB $>255 \mu \mathrm{mol} / \mathrm{L}$.

During the first days after birth, the bilirubin level is changing dynamically, so we use the hour-specific nomogram developed by Bhutani to predict the subsequent severe hyperbilirubinemia [8]. The bilirubin level below the 40th percentile is labeled as a low-risk zone and that above the 95th is labeled as a high-risk zone. Bilirubin levels between 40 to 75 th and 75 th to 95 th percentile are low-intermediate-risk zone and high-intermediate-risk zone, respectively.

The management plan is developed based on the risk zones as follows:

1. TCB level $<75$ th percentile (low-risk or low-intermediate-risk zone): the risk of subsequent significant hyperbilirubinemia is low; infants just need routine care and repeated TCB in 24 hours.

2. TCB level between 75 and 95 th percentile (high-intermediate-risk zone) without any risk factors: the risk of subsequent significant hyperbilirubinemia is still low; infants just need routine care and repeat TCB in 24 hours.

3. TCB level between 75 and 95 th percentile (high-intermediate-risk zone) with at least one of the risk factors: the risk of subsequent significant hyperbilirubinemia is high; infants need aggressive breast feeding, ensure adequate intake and reduce the enterohepatic circulation. TCB should be repeated in 12 hours. Evaluate the causes of jaundice.

4. TCB level $>95$ th percentile (high-risk zone): the risk of subsequent significant hyperbilirubinemia is very high; TSB should be checked. Infants need prompt intervention, start phototherapy, or exchange transfusion if the criteria met. Monitor the bilirubin level closely and evaluate the causes of jaundice.

\section{Parenting education}

With the rapid development of internet technology, smartphone application software (APP) has been used successfully in the monitoring for transcutaneous bilirubin level of newborn infants $[9,10]$. We use online training instead of the classroom teaching during the epidemic. Parents can access the resources including education videos and training brochures on social media. Every parent should be fully informed of the potential harm of hyperbilirubinemia, the importance of bilirubin monitoring, the availability of online resources, and methods for assessing a newborn infant's condition. In addition, detailed information about COVID-19 and prevention strategies should be provided.

\section{Discharge}

\section{Indications of discharge}

Healthy newborn infants with gestational age at 35 or more weeks are usually discharged on days 2 or 3 if the vital signs are stable, if feeding is good and daily weight loss is less than $3 \%$ of birth weight, and if the value of TCB on the 
day of discharge is below the 75th percentile. For the newborn infants admitted to the special care nursery or neonatal intensive-care unit, the indications of discharge include the following: (1) the underlying disease is cured or significantly improved; (2) the vital signs are stable; (3) infants who had been treated with phototherapy and/or exchange transfusion should be observed for at least 24 to 48 hours to make sure of no obvious rebound of bilirubin; (4) the TCB value on the day of discharge is below the 75th percentile.

\section{Follow-up strategy}

The follow-up plan is made based on the gestational age of infants, days after birth, and the bilirubin level on discharge day. The time schedule of the first online follow-up is listed in Table 1. Parents should be advised to use the APP software for remote follow-up and online consultation. Doctors will give the color calibration card for transcutaneous bilirubin measurement to parents. The remote follow-up route is mainly through Internet hospitals or other qualified Internet platforms. Under the guidance of healthcare workers, parents can make an appointment in Internet hospital before infants discharged home.

\section{Remote follow-up after discharge}

\section{General condition evaluation}

The parents shall fill in the questionnaire on the APP software, including the general conditions of the infant, feeding issues, weight changes, abnormal clinical symptoms, etc. (Table 2).

\section{Evaluation of risks of jaundice}

Parents can download and install the APP software on the smart phone for remote follow-up and bilirubin monitoring. Parents may practice using the software under the direction of medical staff. After downloading and installing the APP on a smartphone, parents can place a color calibration

Table 1 Time schedule of online follow-up after discharge

\begin{tabular}{lll}
\hline Age at discharge (h) & $\begin{array}{l}\text { Bilirubin level at dis- } \\
\text { charge (percentile) }\end{array}$ & $\begin{array}{l}\text { Timing for the first } \\
\text { online follow-up }\end{array}$ \\
\hline $48-72$ & $<40$ th & 2-3 d after discharge \\
& 40th-75th & $1-2$ d after discharge \\
$72-96$ & $<40$ th & $3-5$ d after discharge \\
& 40th-75th & $2-3$ d after discharge \\
$96-120$ or more & $<40$ th & $3-5$ d after discharge \\
& 40th-75th & $2-3$ d after discharge \\
\hline
\end{tabular}

card (a modified Macbeth Color Checker card with a small hollow square area) on the skin over the sternum of the newborn, then start the APP, and take a photo of the card by smartphone camera. The image data will be transmitted to the cloud for analysis, the results of transcutaneous bilirubin level will be given automatically in several seconds and will be forwarded to the associated medical staff's smartphone simultaneously. According to the time schedule in Table 1, parents check the TCB level by smartphone as described above. After getting the results, the neonatologists would give advice to parents based on the newborn's situation and the TCB level. If the measured TCB level is variable, parents should recheck it under good lighting condition. If an alarm value is indicated by the software, parents should take the neonates to the hospital. The frequency of bilirubin measurement can be increased at any time if parents feel their infant's jaundice is getting more significant.

\section{Vaccination recommendation}

The WHO vaccination guidelines did not regard neonatal jaundice as the contraindication of vaccination, and there was no clinical evidence that high bilirubin level is an effect of vaccination. However, during the period of epidemic, we suggest that routine vaccination be postponed according to the experts' consensus [11].

\section{Out-patient clinic visiting}

\section{Indications}

During the online follow-up, if any of the following criteria are met, the newborn infant should be taken to the

Table 2 Questionnaire for online follow-up evaluation

\begin{tabular}{ll}
\hline Contents \\
\hline General situations & 1. What is baby's temperature today? \\
& 2. The change of weight \\
3. How many wet diapers changing per day? & 4. Less or very low crying \\
5. High or pitched crying \\
6. Color of lips (blue/purple/pale) \\
7. Any labored breathing? \\
8. Is there any abnormal movements? \\
1. TCB level (via the smartphone APP) \\
2. Degree of jaundice assessed by observation \\
(Involving the head, trunk, limbs, palm) in \\
natural light ${ }^{\mathrm{a}}$ \\
1. Breast feeding or formula feeding? \\
2. The frequency of feeding per day \\
3. Vomiting or not
\end{tabular}

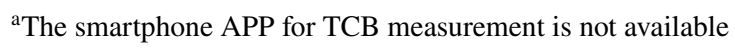


hospital as soon as possible: (1) inadequate milk intake and lethargy; (2) the weight has not return to birth weight by 7-10 days of age; (3) presentation of any other symptoms, such as dyspnea, fever or hypothermia, frequent vomiting, seizure, etc.; (4) the bilirubin level $>95$ th percentile in the first week after birth or more than $255 \mu \mathrm{mol} / \mathrm{L}(15 \mathrm{mg} / \mathrm{dL})$ after 7 days of age, or the increment of bilirubin level is beyond $5 \mathrm{mg} / \mathrm{dL}$ within $24 \mathrm{~h}$; (5) the doctors in charge of the online follow-up suggesting need of an out-patient visit.

\section{Prevention strategies for infants visiting the clinic}

During the epidemic, we should pay high attention to the prevention and control of COVID-19 infection. For each infant visiting the clinic, we should collect the epidemiological history of the infant, the families, caregivers, and visitors during the previous 14 days and we also should measure the body temperature routinely.

Infants can be admitted to the special nursery if without any close contact history with an infected person including family members, caregivers, and visitors. For infants with significant hyperbilirubinemia, we recommend intervention after admission, as the out-patient daytime phototherapy will increase the risk of SARS-CoV-2 infection.

If the infant, family member or caregivers are exposed to any infected person, the infant should be considered as suspected case of infection and should be admitted to a single isolation room with protection. The infant should be settled in an incubator instead of a radiant warmer.

If the infant is critically ill or if the hospital has not been equipped with an isolation unit, emergency transport should be launched. The transport should be organized by the public health administrative department. The transport team should include a physician, nurse, and ambulance driver. All team members should be well trained with the knowledge and skills about COVID-19 infection prevention [11].

Author contributions MXL and CZ contributed equally to this paper. All authors take part in drafting the paper and approved the final version.

Funding: This paper was supported by Zhejiang University Special Scientific Research Fund for COVID-19 Prevention and Control.

\section{Compliance with ethical standards}

Ethical approval Not required.

Conflict of interest The authors have no financial relationships relevance to the article to disclose.

\section{References}

1. Zhu N, Zhang D, Wang W, Li X, Yang B, Song J, et al. Coronavirus investigating, and research team A novel coronavirus from patients with pneumonia in China, 2019. N Engl J Med. 2020. https://doi.org/10.1056/NEJMoa2001017.

2. Huang C, Wang Y, Li X, Ren L, Zhao J, Hu Y, et al. Clinical features of patients infected with novel coronavirus in Wuhan, China. Lancet. 2020. https://doi.org/10.1016/S0140-6736(20)30183-5.

3. Shen KL, Yang YH, Wang TY, Zhao DC, Jiang Y, Jin RM, et al. Diagnosis, treatment, and prevention of novel coronavirus infection in children: experts' consensus statement. World J Pediatr. 2020. https://doi.org/10.1007/s12519-020-00343-7.

4. Chen HJ, Guo JJ, Wang C, Luo F, Yu XC, Zhang W, et al. Clinical characteristics and intrauterine vertical transmission potential of COVID-19 infection in nine pregnant women: a retrospective review of medical records. Lancet. 2020. https://doi.org/10.1016/ s0140-6736(20)30360-3.

5. Zhu HP, Wang L, Fang CZ, Peng SC, Zhang LH, Chang GP, et al. Clinical analysis of 10 neonates born to mothers with 2019nCoV pneumonia. Transl Pediatr. 2019. https://doi.org/10.21037 /tp.2020.02.06.

6. Du LZ, Ma XL. International perspectives: hyperbilirubinemia and kernicterus in neonates in China. NeoReviews. 2012;13:e141-e144144.

7. American Academy of Pediatrics Subcommittee on Hyperbilirubinemia. Management of hyperbilirubinemia in the newborn infant 35 or more weeks of gestation. Pediatrics. 2004;114:297-316.

8. Bhutani VK, Johnson L, Sivieri EM. Predictive ability of a predischarge hour-specific serum bilirubin for subsequent significant hyperbilirubinemia in healthy term and near-term newborns. Pediatrics. 1999;103:6-14.

9. Taylor JA, Stout JW, de Greef L, Goel M, Patel S, Chung EK, et al. Use of a smartphone app to assess neonatal jaundice. Pediatrics. 2017; 140:e20170312

10. Rong ZH, Luo F, Ma LY, Chen L, Wu L, Liu W, et al. Evaluation of an automatic image-based screening technique for neonatal hyperbilirubinemia. Zhonghua Er Ke Za Zhi. 2016;54:597-600 (in Chinese).

11. Wang L, Shi Y, Xiao T, Fu J, Feng X, Mu D, et al. Chinese expert consensus on the perinatal and neonatal management for the prevention and control of the 2019 novel coronavirus infection (First edition). Ann Transl Med 2020;8(3):47.

Publisher's Note Springer Nature remains neutral with regard to jurisdictional claims in published maps and institutional affiliations. 